\title{
History of diabetes mellitus is associated with elevated cardiac troponin I levels in patients with chest pain but no coronary heart disease
}

\author{
Bin Zhong, Yazhu Wang, Guo Zhang
}

Department of Cardiology, The Fifth People's Hospital, Chongqing, China

\begin{abstract}
Background: The factors and the mechanism contributing to an increase in cardiac troponin I (cTnI) in patients with chest pain, at least one cardiovascular risk factor, and no evidence of coronary heart disease remains elusive.
\end{abstract}

Methods: Excluding patients with acute coronary syndrome and chronic myocardial ischemia, we selected 362 consecutive patients with normal coronary angiography or computed tomography coronary angiography results or lesions causing $<50 \%$ stenosis in any of the coronary arteries from January 2012 to June 2015. Using a cut-off value of $0.01 \mathrm{ng} / \mathrm{mL}$, patients with cTnI levels $\geq 0.01 \mathrm{ng} / \mathrm{mL}$ (164 patients) were compared with those with cTnI levels $<0.01 \mathrm{ng} / \mathrm{mL}$. Logistic regression analysis was used to evaluate associations between elevated cTnI and patient characteristics.

Results: Other than history of diabetes mellitus (DM) (18.90\% vs. $8.08 \%, p=0.002)$, the characteristics of the patients with and without elevated cTnI levels were similar. History of DM predicted elevation of $c$ TnI level (OR 3.34, 95\% CI 1.55-7.20, $p=0.002)$ in logistic regression analysis.

Conclusions: In total, $45.30 \%$ of patients with chest pain had elevated cTnI levels with a mean level of $0.07 \pm 0.10 \mathrm{ng} / \mathrm{mL}$. History of DM rather than blood glucose level itself was associated with elevated cTnI levels, whereas female gender was protective against increases in cTnI levels. (Cardiol J 2016; 23, 2: 149-154)

Key words: diabetes mellitus, chest pain, cardiac troponin I, risk factors

\section{Introduction}

Chest pain is a common complaint, usually a symptom of benign conditions. However, some patients with chest pain have serious or lifethreatening diseases such as acute myocardial infarction. The detection of cardiac troponin I (cTnI), especially by point-of-care testing [1-3], is an immediate and effective triage method, not only for rapid risk stratification but also for the prediction of prognosis. Usually, coronary angiography
(CAG) or computed tomography coronary angiography (CTCA) is used to establish final diagnosis; unfortunately, some patients have non-significant stenotic lesions that appear normal on CTCA and CAG despite elevated cTnI levels, inconsistent with acute coronary syndrome. Diagnosis and management in such patients are challenging for physicians. We recruited a cohort of such patients at the cardiology department of our hospital to evaluate the associations between cTnI point-ofcare testing results and patient characteristics. The

Address for correspondence: Bin Zhong, MD, Department of Cardiology, The Fifth People's Hospital, Renji road No. 24 Nanan District, Chongqing, China, fax: +86-023-62896109, e-mail: 13808341072@163.com 
aim of this study was to provide clinical physicians with information to help with the elimination of confounding factors and the correct management of this specific group of patients.

\section{Methods}

\section{Study population and exclusion criteria}

The study population comprised all consecutively hospitalized patients with chest pain, aged $>18$ years and who had at least one cardiovascular risk factor, had undergone CAG or CTCA with normal results, and had lesions causing $<50 \%$ stenosis in any one of the coronary arteries on CAG or CTCA. All patients were recruited from our cardiology department from January 2012 to June 2015. The clinicians decided to test cTnI levels according to current guidelines [4] within $24 \mathrm{~h}$ after admission using the ReLIA SSJ-2 device (Bi-directional Lateral Flow Immunoassay, ReLIA Biotechnologies Co. Ltd., Shenzhen, China). The professional staff ensured quality control. All collected samples were centrifuged and tested immediately when room temperature was reached. A cut-off value of $0.01 \mathrm{ng} / \mathrm{mL}$ was used, and patients with cTnI levels $\geq 0.01 \mathrm{ng} / \mathrm{mL}$ were included in the study group; in contrast, patients with cTnI levels $<0.01 \mathrm{ng} / \mathrm{mL}$ were allocated to the control group. Creatinine clearance $(\mathrm{CC})$ was calculated using the following formula: $(140-$ age $) \times$ body weight $(\mathrm{kg}) \div[0.814 \times$ serum creatinine $(\mu \mathrm{mol} / \mathrm{L})]$ $($ female $\times 0.85)$.

\section{Exclusion criteria}

Patients with the following were excluded:

1. Known coronary heart disease (CHD) and history of coronary revascularization; 2 . Typical exertional chest pain; 3 . Dynamic changes on electrocardiograms; 4 . Dynamic changes in cTnI levels (change of $\geq 20 \%$ at different times within $3 \mathrm{~h}$ to $6 \mathrm{~h}$ after admission); 5. Myocarditis, pericarditis, pleuritis, serious cardiac arrhythmias (exception: patients who had atrial fibrillation and a ventricular rate between 60 to $100 \mathrm{bpm}$ ), hypotension, serious anemia or infection, pulmonary embolism, serious hepatic (liver function - transaminase $\geq 2$ times of the upper normal limit) or renal (results of renal function including $\mathrm{CCs}$ were consistent with the criteria of chronic kidney disease 2 stage or more) dysfunction, malignant tumors, immune system disorders, and hypertriglyceridemia ( $\geq 5 \mathrm{mmol} / \mathrm{L}$ ).

General characteristics, New York Heart Association (NYHA) functional classes, hepatic and renal functions, lipid profiles, and blood glucose levels were recorded according to medical records. The diagnosis of hypertension, diabetes mellitus (DM), valvular heart disease, and cardiomyopathy was based on standard guidelines [5-7]. Biochemical parameters were provided by the central laboratory of our hospital. History of DM was confirmed if two or more results of fasting or postprandial blood glucose tests were consistent with DM or if patients had been receiving pharmacologic or non-pharmacologic treatments for diabetes for at least 1 month before admission.

This study protocol was approved by the Ethics Committee of the Fifth People's Hospital of Chongqing, and all patients provided written informed consent prior to enrollment.

\section{Statistical analysis}

Continuous variables were expressed as means \pm standard deviations (SD), and categorical variables were expressed as frequencies or percentages. Independent samples $t$-tests and $\chi^{2}$ tests were used to analyze differences between groups for continuous variables and categorical variables, respectively. Logistic regression $\chi^{2}$ and Hosmer-Lemeshow tests) was performed to estimate the goodness of fit. Statistical analyses were performed with SPSS version 15.0 (SPSS Inc., Chicago, IL). For all comparisons, a 2 -sided p-value of $<0.05$ was considered statistically significant. When appropriate, 95\% confidence intervals (CI) were calculated.

\section{Results}

Patients with stenotic lesions had more cardiovascular risk factors but similar cTnI levels compared to patients without lesions

We included a total of 362 patients with a mean age of $61.48 \pm 10.52$ years in the analysis, of whom 207 (57.18\%) were women and 194 had non-significant stenosis on CAG or CTCA (Fig. 1). In general, patients with stenosis had cTnI levels similar to those in patients without stenosis $(0.03 \pm$ $\pm 0.07 \mathrm{ng} / \mathrm{mL}$ vs. $0.03 \pm 0.08 \mathrm{ng} / \mathrm{mL}, \mathrm{p}=0.73)$, but were older. History of hypertension and DM was more prevalent in patients with stenosis. Moreover, systolic blood pressure, white blood cell (WBC) count, blood glucose level, and NYHA functional class at admission were higher and $\mathrm{CC}$ were lower in patients with stenosis than those without (Table 1 ). 


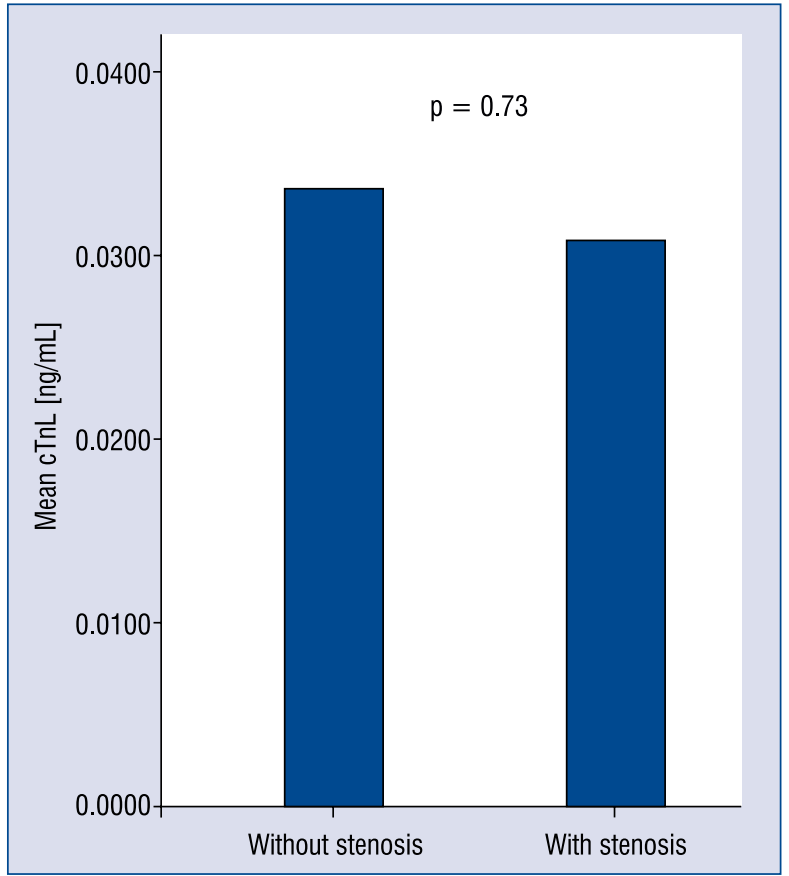

Figure 1. Comparison of cardiac troponin I (cTnl) levels in patients with and without stenosis.
Comparison of clinical parameters in patients with and without increased cTnI levels

The elevated cTnI level group included $164 \mathrm{pa}-$ tients with a mean cTnI level of $0.07 \pm 0.10 \mathrm{ng} / \mathrm{mL}$ (Table 2). There were no differences between the two groups with respect to age, sex, history of hypertension, NYHA functional class, blood pressure and blood glucose level on admission, WBC count, hemoglobin level, blood urea nitrogen level, serum creatinine level, CC, lipid level, and the presence of stenosis on CAG or CTCA. However, history of DM was more prevalent in patients with elevated cTnI levels $(\mathrm{p}<0.01)$.

\section{Analysis of correlations between elevated cTnI and cardiovascular risk factors}

Taking cTnI as a dependent variable and all the clinical parameters as independent variables, binary classification logistic regression was performed to analyze the associations between elevated cTnI and cardiovascular risk factors (Hosmer-Lemeshow goodness of fit test and $\chi^{2}$

Table 1. Comparison of clinical parameters between the patients with and without stenosis.

\begin{tabular}{|c|c|c|c|}
\hline & $\begin{array}{l}\text { Patients with stenosis } \\
\qquad(\mathrm{n}=194)\end{array}$ & $\begin{array}{l}\text { Patients without stenosis } \\
\qquad(\mathrm{n}=168)\end{array}$ & $\mathbf{P}$ \\
\hline Age & $62.85 \pm 10.11$ & $59.89 \pm 10.80$ & $<0.01$ \\
\hline Sex (female) & $112(57.73 \%)$ & $95(56.55 \%)$ & 0.82 \\
\hline History of hypertension & $117(60.31 \%)$ & $70(41.67 \%)$ & $<0.01$ \\
\hline History of DM & $34(17.53 \%)$ & $13(7.74 \%)$ & $<0.01$ \\
\hline NYHA functional class: & & & $<0.01$ \\
\hline I & $161(82.98 \%)$ & $127(75.60 \%)$ & \\
\hline II & $31(16.00 \%)$ & $27(16.07 \%)$ & \\
\hline III & $2(1.03 \%)$ & $13(7.74 \%)$ & \\
\hline IV & $0(0 \%)$ & $1(0.6 \%)$ & \\
\hline $\mathrm{SBP}[\mathrm{mm} \mathrm{Hg}]$ & $141.56 \pm 25.07$ & $134.16 \pm 22.33$ & $<0.01$ \\
\hline $\mathrm{DBP}[\mathrm{mm} \mathrm{Hg}]$ & $83.21 \pm 14.26$ & $80.64 \pm 13.18$ & 0.08 \\
\hline HGB [g/L] & $133.00 \pm 16.61$ & $133.79 \pm 16.21$ & 0.65 \\
\hline WBC $\left[\times 10^{9} / L\right]$ & $6.49 \pm 1.94$ & $6.11 \pm 1.69$ & $<0.01$ \\
\hline BUN [mmol/L] & $5.71 \pm 1.72$ & $5.69 \pm 1.89$ & 0.92 \\
\hline $\mathrm{SCr}[\mu \mathrm{mol} / \mathrm{L}]$ & $72.74 \pm 18.79$ & $70.19 \pm 18.20$ & 0.19 \\
\hline $\mathrm{CCs}[\mathrm{mL} / \mathrm{min}]$ & $79.17 \pm 25.31$ & $85.26 \pm 27.15$ & 0.03 \\
\hline TG $[\mathrm{mmol} / \mathrm{L}]$ & $1.64 \pm 0.83$ & $1.57 \pm 0.89$ & 0.43 \\
\hline LDL [mmol/L] & $2.71 \pm 0.91$ & $2.69 \pm 0.92$ & 0.85 \\
\hline $\mathrm{TC}[\mathrm{mmol} / \mathrm{L}]$ & $4.58 \pm 1.06$ & $4.54 \pm 1.04$ & 0.73 \\
\hline Blood glucose on admission [mmol/L] & $6.69 \pm 2.36$ & $6.20 \pm 1.63$ & 0.02 \\
\hline Percentage of patients with elevation cTnl & 54.9 & 45.1 & 0.66 \\
\hline
\end{tabular}

DM - diabetes mellitus; NYHA - New York Heart Association functional class; SBP — systolic blood pressure; DBP — diastolic blood pressure HGB - hemoglobin; WBC - white blood cell count; BUN - blood urea nitrogen; $\mathrm{SCr}$ - serum creatinine; CCs - creatinine clearances; TG — triglyceride; LDL — low-density lipoprotein cholesterol; TC — total cholesterol; cTnl — cardiac troponin I 
Table 2. Comparison of the clinical parameters of the two groups (with and without elevated cardiac troponin I [cTnl] levels).

\begin{tabular}{|c|c|c|c|}
\hline & $\begin{array}{l}\text { Group with increased } \\
\text { cTnl level }(n=164)\end{array}$ & $\begin{array}{l}\text { Group without increased } \\
\text { cTnl level }(n=198)\end{array}$ & $\mathbf{P}$ \\
\hline Age & $61.41 \pm 10.14$ & $61.55 \pm 10.86$ & 0.90 \\
\hline Sex (female) & $104(63.41 \%)$ & $103(52.02 \%)$ & $<0.05$ \\
\hline History of hypertension & $91(55.49 \%)$ & $96(48.49 \%)$ & 0.18 \\
\hline History of DM & $31(18.90 \%)$ & $16(8.08 \%)$ & $<0.01$ \\
\hline NYHA functional class: & & & 0.25 \\
\hline 1 & $127(76.11 \%)$ & $161(78.51 \%)$ & \\
\hline II & $26(16.81 \%)$ & $32(18.18 \%)$ & \\
\hline III & $10(6.19 \%)$ & $5(3.31 \%)$ & \\
\hline IV & 1 & 0 & \\
\hline $\mathrm{SBP}[\mathrm{mm} \mathrm{Hg}]$ & $138.00 \pm 23.11$ & $138.22 \pm 24.93$ & 0.93 \\
\hline $\mathrm{DBP}[\mathrm{mm} \mathrm{Hg}]$ & $81.86 \pm 12.82$ & $82.15 \pm 14.61$ & 0.84 \\
\hline $\mathrm{HGB}[\mathrm{g} / \mathrm{L}]$ & $131.65 \pm 16.24$ & $134.78 \pm 16.44$ & 0.07 \\
\hline WBC $\left[\times 10^{9} / L\right]$ & $6.41 \pm 2.00$ & $6.24 \pm 1.70$ & 0.38 \\
\hline BUN [mmol/L] & $5.74 \pm 2.00$ & $5.77 \pm 1.62$ & 0.75 \\
\hline $\mathrm{SCr}[\mu \mathrm{mol} / \mathrm{L}]$ & $70.89 \pm 18.88$ & $72.11 \pm 18.27$ & 0.54 \\
\hline $\mathrm{CCs}[\mathrm{mL} / \mathrm{min}]$ & $82.48 \pm 26.47$ & $81.60 \pm 26.26$ & 0.75 \\
\hline $\mathrm{TG}[\mathrm{mmol} / \mathrm{L}]$ & $1.57 \pm 0.83$ & $1.64 \pm 0.88$ & 0.47 \\
\hline LDL [mmol/L] & $2.62 \pm 0.84$ & $2.77 \pm 0.97$ & 0.13 \\
\hline $\mathrm{TC}[\mathrm{mmol} / \mathrm{L}]$ & $4.50 \pm 0.99$ & $4.61 \pm 1.10$ & 0.32 \\
\hline Blood glucose on admission [mmol/L] & $6.49 \pm 2.27$ & $6.61 \pm 1.89$ & 0.68 \\
\hline Presence of stenosis on CAG or CTCA & $90(54.87 \%)$ & $104(52.53 \%)$ & 0.66 \\
\hline
\end{tabular}

CAG - coronary angiography; CTCA — computed tomography coronary angiography; rest abbreviations as in Table 1.

test: 11.17 and $\mathrm{p}=0.19$, respectively) (Table 3 ). Female gender and history of DM were associated with elevated cTnI levels in univariate and multivariate analyses; moreover, history of DM was a risk factor for elevated cTnI levels (odds ratio [OR] 3.34, 95\% CI 1.55-7.20, p < 0.01). In contrast, female gender was a protective factor against elevated cTnI levels (OR $0.48,95 \% \mathrm{CI}$ $0.23-0.99, \mathrm{p}<0.05)$. However, blood glucose level itself on admission did not predict elevated cTnI levels (OR 0.94, 95\% CI $=0.83-1.06, \mathrm{p}=0.31$ ).

\section{Discussion}

Regardless of the presence of stenosis, low cTnI levels were found in $45.30 \%$ of patients with chest paint and at least one cardiovascular risk factor but no evidence of CHD. Patients with stenosis had more risk factors for CHD. History of DM predicted elevated cTnI levels in this cohort, while female gender was a protective factor against increased cTnI level.
As one of the biomarkers of myocardial damage, increased cTnI level is found in many clinical cases without evidence of CHD. To our knowledge, this was the first analysis of the correlations between elevated cTnI level and the characteristics of patients with chest pain with at least one risk factor but no evidence of CHD on CAG or CTCA. Previous studies investigated elevated cTnI levels in various cohorts with different stress responses such as diabetic ketoacidosis and shock [8-10]. However, no studies have included information from CAG or CTGA examinations. Al-Mallah et al. [11] reported that 26 of 96 patients with elevated cTnI levels and diabetic ketoacidosis but no evidence of acute coronary syndrome had higher risks of mortality than patients with normal cTnI levels during a 2-year follow-up (50.0\% vs. $27.1 \%$, HR 2.3, $95 \%$ CI 1.2-4.8, $\mathrm{p}=0.02)$. Mehta et al. [12] found that $16(43 \%)$ of 37 patients with septic shock had elevated cTnI levels ( $\geq 0.01 \mathrm{ng} / \mathrm{mL}$ ). Becattini et al. [10] demonstrated that elevated cTnI levels correlated with short-term mortality (OR 5.24; 95\% CI 
Table 3. Logistic regression analysis of elevated cardiac troponin I (cTnl) and clinical parameters.

\begin{tabular}{|c|c|c|c|c|c|c|c|}
\hline & \multirow[t]{2}{*}{ B } & \multirow[t]{2}{*}{ SE } & \multirow[t]{2}{*}{ Wald } & \multirow[t]{2}{*}{$\mathbf{P}$} & \multirow[t]{2}{*}{$\operatorname{Exp}(B)$} & \multicolumn{2}{|c|}{$95 \%$ confidence interval } \\
\hline & & & & & & Lower & Upper \\
\hline Sex (female) & -0.732 & 0.369 & 3.939 & 0.047 & 0.481 & 0.234 & 0.991 \\
\hline Age & -0.011 & 0.017 & 0.416 & 0.519 & 0.989 & 0.957 & 1.022 \\
\hline History of hypertension & 0.260 & 0.245 & 1.132 & 0.287 & 1.298 & 0.803 & 2.096 \\
\hline History of diabetes & 1.205 & 0.393 & 9.408 & 0.002 & 3.336 & 1.545 & 7.203 \\
\hline Stenosis & 0.048 & 0.235 & 0.042 & 0.838 & 1.049 & 0.662 & 1.663 \\
\hline NYHA class & 0.246 & 0.219 & 1.268 & 0.260 & 1.279 & 0.833 & 1.964 \\
\hline SBP & 0.000 & 0.007 & 0.000 & 0.991 & 1.000 & 0.985 & 1.015 \\
\hline DBP & -0.002 & 0.013 & 0.023 & 0.881 & 0.998 & 0.973 & 1.024 \\
\hline HGB & -0.007 & 0.009 & 0.566 & 0.452 & 0.993 & 0.976 & 1.011 \\
\hline WBC & 0.055 & 0.065 & 0.702 & 0.402 & 1.056 & 0.929 & 1.200 \\
\hline Blood glucose & -0.062 & 0.062 & 1.018 & 0.313 & 0.940 & 0.833 & 1.060 \\
\hline BUN & -0.046 & 0.072 & 0.413 & 0.520 & 0.955 & 0.830 & 1.099 \\
\hline $\mathrm{SCr}$ & 0.014 & 0.012 & 1.402 & 0.236 & 1.014 & 0.991 & 1.038 \\
\hline TG & -0.227 & 0.153 & 2.220 & 0.136 & 0.797 & 0.591 & 1.074 \\
\hline LDL & -0.331 & 0.298 & 1.235 & 0.266 & 0.718 & 0.401 & 1.287 \\
\hline TC & 0.230 & 0.267 & 0.742 & 0.389 & 1.259 & 0.746 & 2.124 \\
\hline CCs & 0.009 & 0.009 & 1.196 & 0.274 & 1.010 & 0.993 & 1.027 \\
\hline
\end{tabular}

Abbreviations as in Table 1.

3.28-8.38) in a meta-analysis. In contrast, based on CAG or CTCA results, we demonstrated that in patients with $<50 \%$ stenosis or normal arteries, $45.30 \%$ patients with no evident stress response had elevated cTnI levels. Of these, $65.96 \%$ of patients with a history of DM had elevated cTnI levels. This was a higher rate than previously reported, possibly because the patients in this analysis had more risk factors than those included in the study by Al-Mallah et al. [11]. Moreover, history of DM was able to predict elevated cTnI levels (OR 3.34, $\mathrm{p}<0.01$ ) in logistic regression analysis, and the risk of elevated cTnI levels in patients with a history of DM was 3-times that of normal patients. It was interesting that blood glucose level at admission could not predict elevated cTnI levels. This phenomenon might reflect, at least in part, the results of randomized control studies such as ACCORD (Cardiovascular Risk in Diabetes trial) [13], which demonstrated that intensive control of blood glucose level itself does not necessarily translate into prognostic benefits other than the microvascular benefits in type $2 \mathrm{DM}$ patients. In this analysis, this may have been due to the fact that blood glucose levels at admission were not significantly higher than normal values $(6.49 \pm$ $\pm 2.27 \mathrm{mmol} / \mathrm{L}$ vs. $6.61 \pm 1.89 \mathrm{mmol} / \mathrm{L})$, in contrast to other studies in which the patients included had diabetic ketoacidosis and markedly higher blood glucose levels than normal. In the previous study, dual effects of hyperglycemia and stress response might have contributed to the elevation of cTnI levels. Unfortunately, we could not analyze DM exclusively due to the limited sample size and lack of integrity of the glycosylated hemoglobin data available.

Various mechanisms might result in cTnI level elevation in patients with DM but no evidence of CHD [14-17], such as diabetic cardiomyopathy, metabolic disturbance, oxidative stress, diabetic microangiopathy, and diabetic nephropathy, although the CCs of all patients were similar in this analysis. Other possible mechanisms include myocardium apoptosis, alteration of myocardium membrane permeability, and myocardial hypertrophy. The multifactorial and comprehensive effects of cardiovascular risk factors were more likely to contribute to elevated cTnI levels. It is reasonable to believe that aggressive treatment of basal diseases and intensive management of all of cardiovascular risk factors including DM is particularly beneficial for these patients, as no standard guidelines have been established and the mechanism of cTnI level elevation in these patients remains elusive. It is impera- 
tive to study this mechanism in patients with no evidence of CHD and normal CAG or CTCA results, as such knowledge might be helpful for clinicians in managing this specific group of patients.

Lack of random controls and the possibility of false negative and positive results for cTnI were the main disadvantages of this analysis. Smoking is one of the traditional risk factors for atherosclerosis, and active smoking and passive smoking have similar hazards, we could not do analysis including this variable because of difficulty for quantification in practice. It is probable that the predictive value of the history of DM or other risk factors could be improved by analyzing a larger patient cohort. We hope that future randomized controlled studies in larger cohorts will help confirm these results.

\section{Conclusions}

Many patients with chest pain, at least one cardiovascular risk factor, and no evidence of $\mathrm{CHD}$ on CAG or CTCA presented with slightly elevated cTnI levels. We found that history of DM was associated with increased cTnI levels, while female gender was a protective factor against increases in cTnI level in this specific population.

\section{Acknowledgments}

We thank colleagues from the Department of Cardiology for their support.

\section{Conflict of interest: None declared}

\section{References}

1. Hjortsh $\varnothing \mathrm{j}$ S, Venge P, Ravkilde J. Clinical performance of a new point-of-care cardiac troponin I assay compared to three laboratory troponin assays. Clin Chim Acta, 2011; 412: 370-375. doi: 10.1016/j.cca.2010.11.015.

2. Kim TK, Oh SW, Hong SC, Mok YJ, Choi EY. Point-of-care fluorescence immunoassay for cardiac panel biomarkers. J Clin Lab Anal, 2014; 28: 419-427. doi: 10.1002/jcla.21704.

3. Renaud B, Maison P, Ngako A et al. Impact of point-of-care testing in the emergency department evaluation and treatment of patients with suspected acute coronary syndromes. Acad Emerg Med, 2008; 15: 216-224. doi: 10.1111/j.1553-2712.2008.00069.x.

4. Amsterdam EA, Wenger NK, Brindis RG et al. 2014 AHA/ACC Guideline for the Management of Patients With Non-ST-Ele- vation Acute Coronary Syndromes: A Report of the American College of Cardiology/American Heart Association Task Force on Practice Guidelines. J Am Coll Cardiol, 2014; 64: e139-e228. doi: 10.1016/j.jacc.2014.09.017.

5. Mancia G, Fagard R, Narkiewicz K et al. 2013 ESH/ESC guidelines for the management of arterial hypertension: The Task Force for the Management of Arterial Hypertension of the European Society of Hypertension (ESH) and of the European Society of Cardiology (ESC). Eur Heart J, 2013; 34: 2159-2219. doi: 10.1093/eurheartj/eht151.

6. Nishimura RA, Otto CM, Bonow RO et al. 2014 AHA/ACC guideline for the management of patients with valvular heart disease: A report of the American College of Cardiology/American Heart Association Task Force on Practice Guidelines. J Am Coll Cardiol, 2014; 63: e57-e185. doi: 10.1016/j.jacc.2014.02.537.

7. Cardiovascular Epidemiology Branch of The Republic Of China. The cardiovascular disease magazine editorial board. Cardiomyopathy diagnosis and treatment recommendations. Chin J Cardiol, 2007; 35: 5-16.

8. De Marco T, Fonarow GC, Diercks D, Diercks D, Wynne J. Cardiac troponin and outcome in acute heart failure. $\mathrm{N}$ Engl J Med, 2008; 358: 2117-2126. doi: 10.1056/NEJMoa0706824.

9. Meyer T, Binder L, Hruska N, Luthe H, Buchwald AB. Cardiac troponin I elevation in acute pulmonary embolism is associated with right ventricular dysfunction.J Am Coll Cardiol, 2000; 36: 1632-1636.

10. Becattini C, Vedovati MC, Agnelli G. Prognostic value of troponins in acute pulmonary embolism: A meta-analysis. Circulation, 2007; 116: 427-433.

11. Al-Mallah M, Zuberi O, Arida M, Kim HE. Positive troponin in diabetic ketoacidosis without evident acute coronary syndrome predicts adverse cardiac events. Clin Cardiol, 2008; 31: 67-71. doi: 10.1002/clc.20167.

12. Mehta NJ, Khan IA, Gupta V, Jani K, Gowda RM, Smith PR. Cardiac troponin I predicts myocardial dysfunction and adverse outcome in septic shock. Int J Cardiol, 2004; 95: 13-17.

13. Ismail-Beigi F, Craven T, Banerji MA et al. Effect of intensive treatment of hyperglycaemia on microvascular outcomes in type 2 diabetes: An analysis of the ACCORD randomised trial. Lancet, 2010; 376: 419-30. doi: 10.1016/S0140-6736(10)60576-4.

14. American Diabetes Association. Standards of medical care in diabetes: 2010. Diabetes Care, 2010; 33: S11-S61. doi: 10.2337/ dc10-S011.

15. Preedy V. Diabetic cardiomyopathy and oxidative stress. In: Diabetes Oxidative Stress and Dietary Antioxidants. Academic Press is an imprint of Elsevier; 2014: 26-32.

16. Newby LK, Jesse RL, Babb JD et al. ACCF 2012 expert consensus document on practical clinical considerations in the interpretation of troponin elevations: A report of the American College of Cardiology Foundation task force on Clinical Expert Consensus Documents. J Am Coll Cardiol, 2012; 60: 2427-2463. doi: 10.1016/j.jacc.2012.08.969.

17. Kociol RD, Pang PS, Gheorghiade M et al. Troponin elevation in heart failure prevalence, mechanisms, and clinical implications. J Am Coll Cardiol, 2010; 56: 1071-1078. doi: 10.1016/j. jacc.2010.06.016. 